\title{
A New Regime of Anomalous Penetration of Relativistically Strong Laser Radiation into an Overdense Plasma
}

\author{
A. Kim, ${ }^{1}$ F. Cattani, ${ }^{2}$ D. Anderson, ${ }^{2}$ and M. Lisak ${ }^{2}$ \\ ${ }^{1}$ Institute of Applied Physics, Russian Academy of Sciences, 603600 Nizhny Novgorod, Russia \\ ${ }^{2}$ Department of Electromagnetics, Chalmers University of Technology, S-412 96 Göteborg, \\ Sweden
}

\begin{abstract}
It is shown that penetration of relativistically intense laser light into an overdense plasma, accessible by self-induced transparency, occurs over a finite length only. The penetration length depends crucially on the overdense plasma parameter and increases with increasing incident intensity after exceeding the threshold for self-induced transparency. Exact analytical solutions describing the plasma-field distributions are presented.

PACS number(s): 52.40.Nk, 52.35.Mw, 52.60.+h, 52.58.Ns
\end{abstract}

In the past few years there has been much research devoted to the nonlinear interaction of superintense laser pulses with plasmas [1]. At intensities where electrons quiver with relativistic velocities, the interaction can be characterized as nonlinear optics in relativistic plasmas, and new regimes, not evident at nonrelativistic intensities, may appear. As was previously shown, superintense electromagnetic radiation can propagate through a classically overdense plasma due to the relativistic correction to the electron mass, the so called induced transparency effect, [2]- [10]. The present work has resulted in the identification of a new fundamental process in relativistic laser overdense plasma interaction.

In order to understand the nonlinear regime of interaction of superintense laser light with an overdense plasma, it is enough, without loss of generality, to consider a stationary model. We present here a new class of exact analytical solutions describing the penetration of an electromagnetic wave normally incident onto a cold, overdense plasma with a sharp boundary. In particular, we show that, when the incident intensity exceeds the threshold for self-induced transparency, the laser energy penetrates into the dense plasma without any losses, but over a finite length only. At the same time, the electron density distribution becomes structured as a sequence of electron layers separated by about half a wavelength wide depleted regions, so that this strongly nonlinear plasma structure acts as a distributed Bragg reflector.

The ultrahigh intensity laser-plasma interaction is described by the relativistic equation of motion and the equation of continuity for the electrons together with Maxwell's equations. Ions are treated as a uniform neutralizing background. We will consider circularly polarized laser radiation with normalized amplitude of the vector potential $e \mathbf{A} / m c^{2}=(a / \sqrt{2}) \operatorname{Re}[(\mathbf{y}+$ $i \mathbf{z}) \exp (i \omega t)]$ normally incident from vacuum $(x<0)$ onto a semi-infinite plasma $(x \geq 0)$. Assuming a stationary regime, the basic equations may be written in the form

$$
\begin{aligned}
& \phi^{\prime \prime}=n_{o}(n-1), \\
& a^{\prime \prime}+\left(1-\frac{n_{o}}{\gamma} n\right) a=0, \\
& \phi^{\prime}=\gamma^{\prime} \text { if and only if } n(x) \neq 0 .
\end{aligned}
$$

(where variables are normalized as $x \Rightarrow \omega x / c, n \Rightarrow n / n_{o}, n_{o}=\omega_{p}^{2} / \omega^{2}, \omega$ is the carrier frequency of the laser radiation, $\omega_{p}$ is the plasma frequency of the initial unperturbed plasma, $\gamma=$ $\left(1+a^{2}\right)^{1 / 2}$ is the relativistic factor, $n$ and $\phi$ are normalized electron density and scalar potential 
of the plasma respectively). Eq. (3) indicates that only in the region where the electron density $n(x) \neq 0$ the ponderomotive force, $\gamma^{\prime}(x)$, must be compensated by the force of a longitudinal field. This statement will be important for constructing solutions of interest in the present analysis.

For homogeneous ion density, the system has the following Hamiltonian

$$
\mathcal{H}_{E}=\frac{1}{2\left(1+a^{2}\right)} a^{\prime 2}-\frac{1}{2}\left(2 n_{o} \sqrt{1+a^{2}}-a^{2}\right)
$$

which was analyzed in [5].

As we are interested in a semi-infinite plasma, we first consider the case when $n(x) \rightarrow 1$ and both $a(x)$ and $a^{\prime}(x)$ vanish as $x \rightarrow \infty$. In this case the integral of motion equals

$$
\mathcal{H}_{E}=-n_{o} \equiv \mathcal{H}_{0}
$$

Eq. (4) can then easily be integrated to yield the following single-parametric solitary solution

$$
a(x)=\frac{A_{m} \cosh \left[\left|\varepsilon_{0}\right|^{1 / 2}\left(x-x^{(0)}\right)\right]}{n_{o} \cosh ^{2}\left[\left|\varepsilon_{0}\right|^{1 / 2}\left(x-x^{(0)}\right)\right]-\left|\varepsilon_{0}\right|}
$$

where $\varepsilon_{0}=1-n_{o}$ is the dielectric permittivity of the plasma and the parameter $x^{(0)}$ defines the position of the maximum of the function (6) which is given by $A_{m}=2\left[n_{o}\left(n_{o}-1\right)\right]^{1 / 2}$.

For $n_{o}>1.5$ this solution contains a region where the electron density is negative, which is clearly unphysical. Requiring the minimum electron density $n_{m}=1-4\left(n_{o}-1\right)^{2}$ to be positive, i.e. $n_{m} \geq 0$, we obtain a condition on the background plasma density and an argument in favor of the depletion region: for $n_{o}>1.5$ we have to take into account only that part of the solution where the corresponding electron density is positive, $n(x) \geq 0$. By using the part of the solution (6) corresponding to a ponderomotive force that pushes electrons into the plasma and matching it to the vacuum one, an exact expression for the intensity threshold of self-induced transparency was found, [1]. It should be noted that the Hamiltonian (5) corresponds to a zero energy flux, but there are solutions with non-zero flux as well, [2, 6]. They can arise even in correspondence to incident amplitudes smaller than the penetration threshold. However, for the realization of such nonlinear hysteresis-like solutions, [10, 12, preliminary modifications of the plasma must be induced by extremely intense fields. For the description of the steady-state of a problem involving laser radiation with turn-on shape it is natural to choose the zero flux approach.

The left side of the function (6) gives rise to an unbalanced ponderomotive force pulling electrons out of the plasma towards the incident wave and, at first glance, it seems that the charge quasineutrality condition cannot be satisfied. However, after a length of about half a wavelength, these electrons will be stopped by the ponderomotive force acting in the opposite direction. Thus, in the general case, we may expect that the full plasma-field structure will consist of a sequence of alternating depletion and nondepletion regions. This can be understood from Fig. 1, where the phase portraits described by Eqs (1)-(3)and by the corresponding equation for the depletion (vacuum) region are presented. For a half-space plasma the limiting case corresponds to a motion with infinite period along the separatrix determined by Eq. (5), i.e., an exponentially decreasing field inside the overdense plasma. Going backwards towards the initial vacuum-plasma boundary, before the last semi-infinite electron layer there must exist 
a depletion region. Here the amplitude of the field corresponds to a forward going wave along the incident direction with an intensity below the threshold value (corresponding to the motion along the first circular trajectory in phase space, coming from the vacuum Hamiltonian). Then, in front of this layer we have to put another electron layer, where the solution for the field $a(x)$ follows from the Hamiltonian (4) with a magnitude $\mathcal{H}>-n_{o}$ (corresponding to an oscillatory motion about zero). This construction is repeated until the initial plasma boundary is reached. At the boundary between each depletion and nondepletion region, the solution for the field must satisfy continuity conditions for both $a$ and its derivative $a^{\prime}$. It is also clear that there exists a family of stationary solutions that differ from each other by the number of electron density layers and their shapes. When the incident amplitude is increased, the number of layers will increase as well, as follows from the phase portraits in Fig. 1.

In order to quantify the above discussion, we present a more rigorous analytical description. Starting from inside the plasma region, the solution for the field is an exponentially decreasing function of the spatial coordinate, in fact a part of the localized solution given by Eq. (6) with $\mathcal{H}_{E}=\mathcal{H}_{0}$ for $x_{0} \leq x<\infty$. The point $x=x_{0}$ can be determined self-consistently from the global solution and the boundary conditions

$$
a\left(x=x_{0}\right)=a_{0}, a^{\prime}\left(x=x_{0}\right)=a_{0}^{\prime}
$$

The next region $\left(x_{1}<x<x_{0}\right)$ must be a depletion layer where $n(x)=0$. The second boundary position of this depletion layer, $x_{1}$, must also be determined self-consistently. The Hamiltonian here is the vacuum one and reads

$$
\mathcal{H}_{V}=\frac{1}{2}\left(a^{\prime 2}+a^{2}\right)=\frac{1}{2}\left(a_{0}^{\prime 2}+a_{0}^{2}\right) \equiv \mathcal{H}_{1}
$$

where we have taken into account the boundary conditions for the field and its first derivative. The solution of the field in this depletion region reads

$$
a(x)=A_{1} \cos \left(x+\psi_{1}\right) .
$$

Using (7) we have: $A_{1}=\left(a_{0}^{\prime 2}+a_{0}^{2}\right)^{1 / 2}=\left(2 \mathcal{H}_{1}\right)^{1 / 2}, \psi_{1}=-\arctan \left(a_{0}^{\prime} / a_{0}\right)-x_{0}$. The boundary position $x_{1}$ can be calculated by integrating Poisson's equation within the interval $x_{1}<x<x_{0}$ :

$$
\phi^{\prime}\left(x_{0}\right)-\phi^{\prime}\left(x_{1}\right)=-n_{o}\left(x_{0}-x_{1}\right) .
$$

which, together with relation (3), leads to a transcendental equation for $\xi=x_{0}-x_{1}$ :

$$
\xi=g(\xi)-g(0)
$$

where

$$
g(\xi)=\frac{A_{1}^{2} \sin \left[2\left(\xi+\xi_{1}\right)\right]}{2 n_{o}\left[1+A_{1}^{2} \cos ^{2}\left(\xi+\xi_{1}\right)\right]^{1 / 2}}, \quad \xi_{1}=\arctan \left(\frac{a_{0}^{\prime}}{a_{0}}\right)
$$

Since the left-hand side of Eq. (11) is a linear function while its right-hand side is a periodic function of $\xi$, this equation has a non-trivial solution only if $g^{\prime}\left(\xi=-\xi_{1}\right)>1$, i.e.

$$
g^{\prime}\left(\xi=-\xi_{1}\right)=\frac{A_{1}^{2}}{n_{o}\left(1+A_{1}^{2}\right)^{1 / 2}}>1
$$

However, if $n_{o} \leq 1.5$, this condition is never satisfied, because its maximum value reaches one at $n_{o}=1.5$. Consequently, for plasma densities $n_{o} \leq 1.5$ we conclude that there are no stationary 
regimes of anomalous penetration: there can only exist dynamical solutions. For $n_{o}>1.5$, Eq. (11) always has non-trivial solutions, which can be found numerically. Formally, Eq. (11) admits several roots but we have to consider only those that correspond to a positive electron density.

Having solved Eq.(11) we know $a_{1}=a\left(x_{1}\right)$ and $a_{1}^{\prime}=a^{\prime}\left(x_{1}\right)$. The next region must again be an electron layer. Denoting this region as $x_{2} \leq x \leq x_{1}$, the Hamiltonian (四) will here be

$$
\mathcal{H}_{E}=\mathcal{H}_{E}\left(a_{1}, a_{1}^{\prime}\right) \equiv \mathcal{H}_{2}
$$

with the corresponding field solution written in terms of two-parameter elliptic functions for the field strength as

$$
a(x)=\left\{\begin{array}{l}
\frac{2 q \operatorname{cn}\left[\varepsilon_{2}^{1 / 2}\left(x-x^{(2)}\right)\right]}{2+\left[\left(q^{2}+1\right)^{1 / 2}-1\right] \operatorname{sn}^{2}\left[\varepsilon_{2}^{1 / 2}\left(x-x^{(2)}\right)\right]}, \mathcal{H}_{2}<n_{o}, \\
\frac{2 \bar{q} \operatorname{sn}\left[\left(\left(\varepsilon_{2}+1\right)^{2}-n_{o}^{2}\right)^{1 / 2}\left(x-x^{(2)}\right) / 2\right]}{\bar{q}^{2}-\operatorname{sn}^{2}\left[\left(\left(\varepsilon_{2}+1\right)^{2}-n_{o}^{2}\right)^{1 / 2}\left(x-x^{(2)}\right) / 2\right]}, \mathcal{H}_{2}>n_{o}
\end{array}\right.
$$

The two parameters, $\varepsilon_{2}$ and $x^{(2)}$ are determined by the boundary conditions at $x=x_{1}$. Here $\varepsilon_{2}=\left(n_{o}^{2}+1+2 \mathcal{H}_{2}\right)^{1 / 2}, q=\left[\left(\varepsilon_{2}+n_{o}\right)^{2}-1\right]^{1 / 2}$ and $\bar{q}=\left[\left(\varepsilon_{2}+n_{o}+1\right) /\left(\varepsilon_{2}+n_{o}-1\right)\right]^{1 / 2}$, $k=\left[\left(n_{o}^{2}-\left(\varepsilon_{2}-1\right)^{2}\right) / 4 \varepsilon_{2}\right]^{1 / 2}$ and $\left.\left.\bar{k}=\left[\left(\varepsilon_{2}-1\right)^{2}-n_{o}^{2}\right) /\left(\varepsilon_{2}+1\right)^{2}-n_{o}^{2}\right)\right]^{1 / 2}$ are the moduli of the elliptic integrals of the first kind respectively for the two cases. This solution is realized over about a quarter period, where the electron density is positive and is given by

$$
n(x)=\frac{2 \sqrt{1+a^{2}}}{n_{o}}\left(\mathcal{H}_{2}+\frac{3}{2} n_{o} \sqrt{1+a^{2}}-a^{2}\right)
$$

The second boundary position of this layer can now be defined with a certain arbitrariness, namely, it can be taken within the whole interval $x^{*} \leq x_{2} \leq x^{* *}$, where $x^{*}$ is such that $n\left(x^{*}\right)=0$, while $x^{* *}$ is defined by the existence of a solution of the transcendental equation (11). This problem comes up for every layer, therefore the global solution, for a given incident amplitude, in general may not be unique because the thickness of each plasma layer is not uniquely fixed, the realization of each specific solution depending on the prehistory of the process. This is also confirmed by our preliminary simulations based on a hydrodynamics approach where, at fixed incident amplitude, the thickness of the various electron layers in the quasi-stationary stage is quite different depending on the turn-on shape of the incident radiation. The appearance of non-unique solutions is typical of phenomena involving nonlinear media (see, for instance, [12]).

For the sake of concreteness, in what follows, we choose the next boundary position $x_{2}$ as the point where the electron density vanishes, i.e. $n\left(x_{2}\right)=0$. In this case, the transcendental equation (11) always has nontrivial solutions because, if the condition given by Eq. (13) is satisfied for the semi-infinite layer, it will automatically be satisfied for all the precedent electron layers, for the point where the electron density vanishes.

As follows from (16), the field at this boundary is such that

$$
3 n_{o}\left(1+a_{2}^{2}\right)^{1 / 2}=2\left(a_{2}^{2}-\mathcal{H}_{2}\right)
$$

and, making use of the equation for the Hamiltonian (14), we can calculate the first derivative of the field. Thus, for the next depletion layer located within $x_{3}<x<x_{2}$, we know the boundary values of $a_{2}$ and $a_{2}^{\prime}$ that are required to generate a solution by using the same procedure as before for $x_{1}<x<x_{0}$. This procedure is repeated for every layer, $x_{i+1}<x<x_{i}$, up to the final layer and if $\mathcal{H}_{i}>n_{o}$, the field solution has to be taken as in the second expression of (15). 
The last layer will be a depletion region $0<x<x_{N}$ where $x=0$ is the real plasma-vacuum boundary. At this point the electric field due to charge separation must vanish. Integrating Poisson's equation over the plasma interval, the plasma neutrality condition gives

$$
x_{0}=\frac{1}{n_{o}} \frac{a_{N} a_{N}^{\prime}}{\sqrt{1+a_{N}^{2}}}
$$

which defines the last free parameter. Thus, matching the solution for the field to the vacuum solution at the electron layer boundary $x=x_{N}$ we can relate the obtained plasma-field distribution to the incident electromagnetic wave. In this way we can construct an exact stationary solution for the anomalous penetration regime. An example of such a solution is presented in Fig. 2. Notice that the maximum of the electron density in a layer increases from layer to layer, reaching an absolute maximum in the last layer nearest to the vacuum boundary while the width of the layers become more and more narrow. This may easily be understood from Fig. 1; higher lying trajectories have higher values of the integrals of motion. It also means that at higher incident wave amplitudes there are more layers, so that the penetration length will increase with increasing incident intensity. These solutions can naturally be extended to cover the case of plasma slabs with finite thickness, if the penetration length is smaller than the slab thickness.

The following consequence of the previous analysis should be emphasized: For a fixed amplitude the laser field penetrates only a finite length into the overdense plasma. Consequently, if the incident laser pulse has a finite duration, the electromagnetic energy deposited in the plasma will be reflected back into the vacuum after the laser field has vanished. Evidently the transient regime will be more complicated as vacuum (depletion) regions surrounded by electron layers will show a resonator-like behaviour, with the electromagnetic energy being excited by the incident pulse. We expect this field structure to evolve and to be slowly reflected back into the vacuum region.

In conclusion, we have presented a new class of exact analytical stationary solutions describing a new feature of the interaction between a super-intense laser and an overdense plasma. This analysis shows how, depending on the initial plasma density, the interaction might result in the generation of a new plasma-field structure consisting of alternating electron and "vacuum" regions, with the electromagnetic energy penetrating into the overdense plasma over a finite length determined by the incident intensity.

This work was supported in part by the Russian Fund for Fundamental Research (grants No. 98-02-17015 and No. 98-02-17013). One of the authors (F.C.) acknowledges support from the European Community under the contract ERBFMBICT972428.

\section{Captions}

Fig. 1 Phase portrait of the system for $n_{o}=1.6$ and homogeneous ion density, following from Eqs. (4) and (8), see also Fig. 2. The dashed lines stand for vacuum regions, the continuous lines stand for plasma regions, the actual trajectory is given by the thick line and it runs clockwise. The large dashed line denotes the regions where the electron density is negative.

Fig. 2 The continuous line represents the plasma-field structures in a semi-infinite plasma 
initially occupying the region $x \geq 0$, for $n_{o}=1.6$, the unperturbed electron density being represented by the dashed line. The dotted line represents the resulting electron density distribution. All quantities are dimensionless.

\section{References}

[1] S.C. Wilks and W. Kruer, IEEE J. Quantum Electron. 33, 1954, (1997); see also in Superstrong Fields in Plasmas, AIP Conf. Proc. 426, (1998).

[2] A.I. Akhiezer and R.V. Polovin, Sov. Phys. JETP 3, 696, 1956

[3] P.Kaw and J.Dawson, Phys. Fluids 13, 472 (1970).

[4] C.Max and F.Perkins, Phys. Rev. Lett. 27, 1342 (1971).

[5] J.H.Marburger and R.F.Tooper, Phys. Rev. Lett. 35, 1001 (1975).

[6] C. S. Lai, Phys. Rev. Lett. 36, 966 (1976); F. S. Felber and J. H. Marburger, Phys. Rev. Lett. 36, 1176 (1976).

[7] E. Lefebvre and G. Bonnaud, Phys. Rev. Lett. 74, 2002 (1995).

[8] H. Sakagami and K. Mima, Phys. Rev. E 54, 1870 (1996).

[9] S.Guerin, P.Mora, J.C.Adam et al., Phys. Plasma 3, 2693 (1996).

[10] V.V. Goloviznin, T. J. Schep, JETP Lett. 70, 450 (1999)

[11] F. Cattani et al., Phys. Rev. E, in press.

[12] A.G. Litvak, Rev. Plasma Phys. 10, 294 (1992) 
Fig. 1

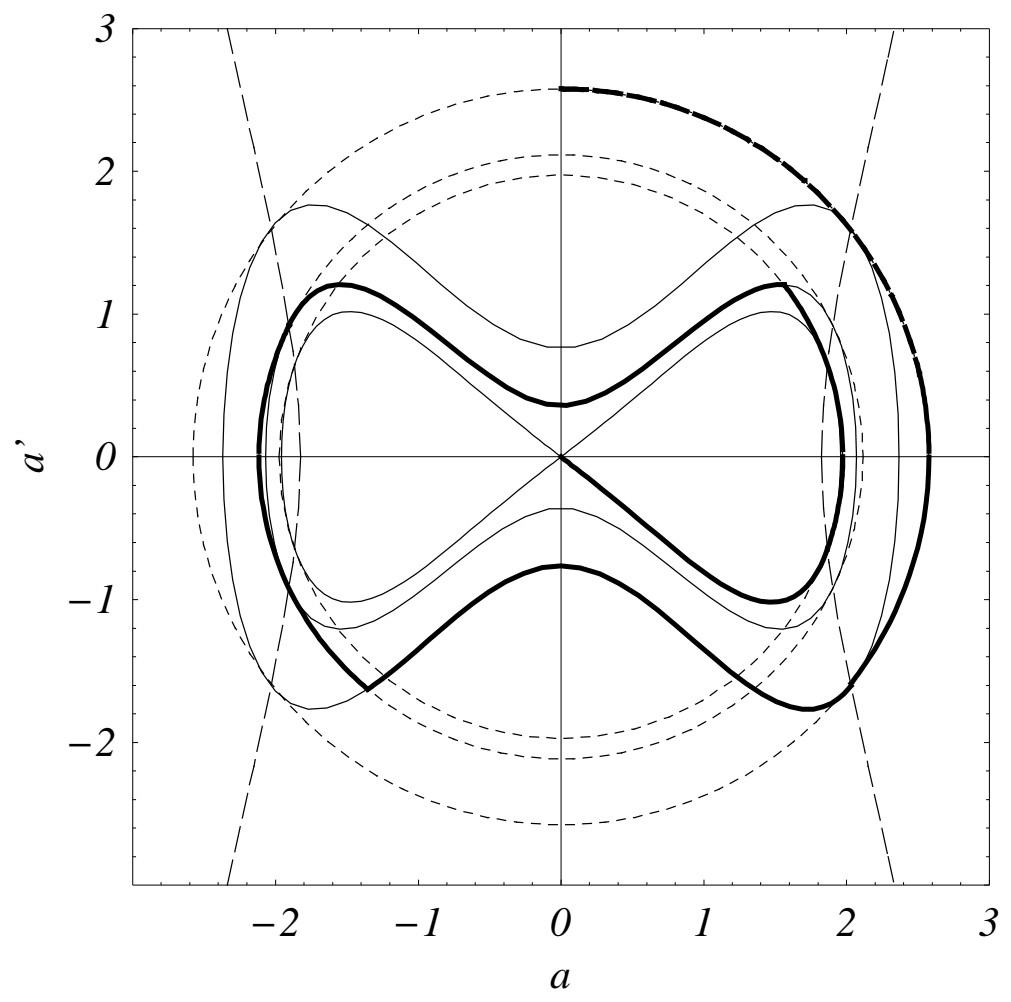


Fig. 2

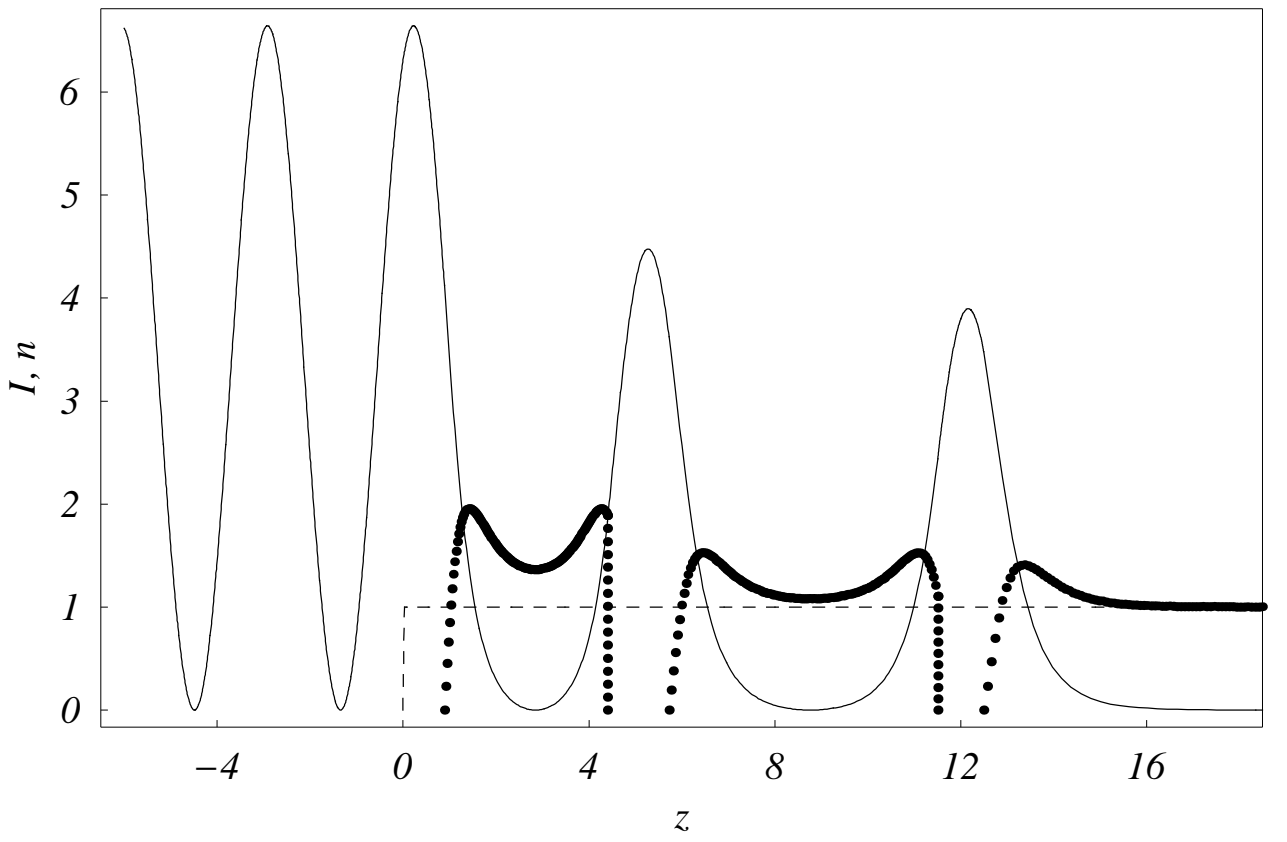

